\title{
Distance between inherent structures and the influence of saddles on approaching the mode coupling transition in a simple glass former
}

\author{
Gabriel Fabricius* \\ Departamento de Fisica, Universidad Nacional de La Plata, cc 67, 1900 La Plata, Argentina \\ Daniel A. Stariolo ${ }^{\dagger}$ \\ Departamento de Fisica, Universidade Federal do Rio Grande do Sul, CP 15051, 91501-970 Porto Alegre, Brazil
}

(Received 11 June 2002; published 10 September 2002)

\begin{abstract}
We analyze through molecular dynamics simulations of a Lennard-Jones (LJ) binary mixture the statistics of the distances between inherent structures sampled at temperatures above the mode coupling transition temperature $T_{\mathrm{MCT}}$. After equilibrating at $T>T_{\mathrm{MCT}}$ we take equilibrated configurations and randomly perturb the coordinates of a given number of particles. After that we find the nearby inherent structures (IS) of both the original and perturbed configurations and evaluate the distance between them. This distance presents an inflection point at $T_{l i} \simeq 1$ with a strong decrease below this temperature which goes to a small but nonzero value on approaching $T_{\mathrm{MCT}}$. In the low-temperature region we study the statistics of events which give zero distance, i.e., dominated by minima, and find evidence that the number of saddles decreases exponentially near $T_{\mathrm{MCT}}$. This implies that saddles continue to exist even at $T \leqslant T_{\mathrm{MCT}}$. As at $T_{\mathrm{MCT}}$ the diffusivity goes to zero, our results imply that there are saddles associated with nondiffusive events at $T<T_{\mathrm{MCT}}$.
\end{abstract}

DOI: 10.1103/PhysRevE.66.031501

PACS number(s): 61.43.Fs, 61.20.Ja, 61.43.-j

What is the influence of the potential energy landscape on the dynamical properties of glass formers? Understanding of this relation is crucial for the development of a comprehensible thermodynamics of supercooled liquids and glasses. In recent years much progress have been done in this direction [1]. Simulating simple models of glass formers Sastry et al. [2] found evidence of four regions where the influence of the landscape is qualitatively different: (a) the high-temperature region where the system is essentially a simple liquid, with exponentially decreasing time correlation functions and free diffusion, (b) a landscape influenced region characterized by the onset of nonexponential relaxation for $T_{\mathrm{MCT}}<T<T_{l i}$, (c) a region which Sastry et al. called landscape dominated in which activated events should be dominant, and (d) the region for $T<T_{g}$ corresponding to the glass phase. In this work we will be concerned with the first two regions and the crossover between the second and the third one. In regions (a) and (d) the landscape seen by the system is essentially flat, of course for very different reasons: in (a) the energy of instantaneous configurations is much higher than typical potential energies and in (d) the system is able only to explore a strictly local region mainly through vibrations and diffusion is avoided. In (b) and (c) the ruggedness of the landscape has a strong influence on the dynamics and makes a thermodynamic description very difficult to rationalize. More recently a more quantitative description of the role of the landscape has begun to emerge [3-8]. An interesting way of looking at changes in the landscape topology in the different regions is to calculate the statistics of saddle points. By looking at the order of the saddles, i.e., the number of unstable directions $n_{s}$, Angelani et al. [4] found that this is a well

\footnotetext{
*Electronic address: fabriciu@fisica.unlp.edu.ar

†Electronic address: stariolo@if.ufrgs.br;

URL: http://www.if.ufrgs.br/ stariolo
}

defined function of temperature and found evidence that it goes to zero at a temperature which seems to coincide with the mode coupling transition temperature $T_{\mathrm{MCT}}$ [9]. The immediate consequence of this result is that the mode coupling transition can be interpreted in terms of a topological change in the landscape: $T_{\mathrm{MCT}}$ should mark the transition between a region dominated by saddles to a region dominated by minima. That this is qualitatively true is now reasonably well established but the quantitative identification of $T_{\mathrm{MCT}}$ with the point where $n_{s}$ goes to zero needs to be independently verified for different systems and if possible by looking at several different quantities. One of these is the mean squared distance (MSD). In Ref. [2] the MSD was calculated between an instantaneous configuration and the nearby IS. The main conclusion was that at very low temperatures, typically below $T_{\mathrm{MCT}}$, it goes linearly to zero as $T \rightarrow 0$, an evidence that the MSD comes essentially from harmonic vibrations. In Ref. [4] it was shown the MSD between saddles and the corresponding minima suggesting a linear relation also between this MSD and the order of the saddles $n_{s}$.

Here we look at the distances between near inherent structures which give additional information on the relation between landscape topology and the dynamics of the system. We performed molecular dynamics simulations on a well known Lennard-Jones binary mixture of $80 \%$ particles of type $A$ and $20 \%$ particles of type $B$ with $\epsilon_{A A}=1.0, \epsilon_{B B}$ $=0.5, \epsilon_{A B}=1.5, \sigma_{A A}=1.0, \sigma_{B B}=0.88, \sigma_{A B}=0.8$ at a density of 1.204 that has been extensively studied for 1000 particles by Kob et al. $[10,11]$ who obtained $T_{\mathrm{MCT}} \simeq 0.435$. In the present work we used $N=N_{A}+N_{B}=250$. We first equilibrate the system at temperatures $T=5,4,3,2,1,0.8,0.6$, $0.55,0.5$, and 0.47 . We study 32 samples at each temperature following different thermal treatments in order to have the same statistics than in Ref. [10] and verify that extrapolating our data to lower temperatures the diffusivity should 
go to zero at the same value of $T_{\mathrm{MCT}}$. The distance between IS is calculated as follows: once the system is equilibrated at a temperature $T$ a typical configuration is taken. Then we make a damage on the equilibrated configuration by changing randomly the coordinates of $n_{d}$ particles chosen also randomly and the IS corresponding to the original configuration and the damaged one are obtained via conjugate gradients minimization. The MSD between both IS is defined as

$$
D^{2}(T, d)=\left\langle\sum_{i}\left(\mathbf{r}_{i}^{I S_{0}}-\mathbf{r}_{i}^{I S_{d}}\right)^{2}\right\rangle,
$$

in which $T$ is the temperature at which the system has been equilibrated, $d$ is the amount of damage done to the equilibrated configuration, i.e., the number of particles perturbed and the dislocation per particle and the braces mean an average taken over a number of independent equilibrium configurations and by making different damages on each configuration chosen, for example, by taking different particles to move for each configuration. As we want to explore the distance between near IS, i.e., those accesible dynamically at each temperature, the damage should be small, so we displace each one of the $n_{d}$ particles an amount $\left|\delta \mathbf{r}_{i}\right|=0.01$ or $\left|\delta \mathbf{r}_{i}\right|=0.10$, both much smaller than the typical interparticle distance of the system [12]. Note that the distance $D^{2}$ goes to zero when the inherent structures of the original configuration and the damaged ones are the same, or in other words, when they are in the basin of a minimum, with no double well saddles between them. We found that at low temperatures an important fraction $f_{0}$ of the events gives zero distance, and so in order to get sensible results we normalize $D^{2}$ with the fraction of nonzero events $1-f_{0}$. Doing this, even in the case where only one event gives a nonzero distance, the resulting $D^{2}$ will be finite and equal to this distance. Concerning the number of particles to be damaged $n_{d}$, we have checked that at low temperatures increasing $n_{d}$ from 2 to 40 only improves the statistics increasing $1-f_{0}$ but not changing qualitatively the behavior of $D^{2}(T, d) /\left(1-f_{0}\right)$. So we take $n_{d}=40$ and keep it fixed in the following study, the amount of damage " $d$ " in expression (1) can therefore be fully characterized by the size of the dislocation $|\delta \mathbf{r}|$. For temperatures $T<0.6$ we have taken averages over 1000 configurations for each one of the 32 samples covering a time range greater than the relaxation time at each $T$. Then for each configuration we have also averaged over four and eight different particles damaged for $d=0.10$ and $d=0.01$, respectively.

A natural question is: is there some temperature at which $D^{2}(T, d)$ goes to zero? In this case this should coincide with the point at which the order of the saddles goes to zero as discussed above.

In Fig. 1 it is shown the normalized MSD as a function of temperature for two different damages. As expected the distance is very large at high temperatures and decreases upon decreasing $T$. Between $T=0.8$ and $T=1$ an inflection point is clearly seen below which the MSD decreases very quickly. This temperature range is the same at which Sastry et al. [2] found the onset of nonexponential behavior in the relaxation functions signalling the beginning of what they called the

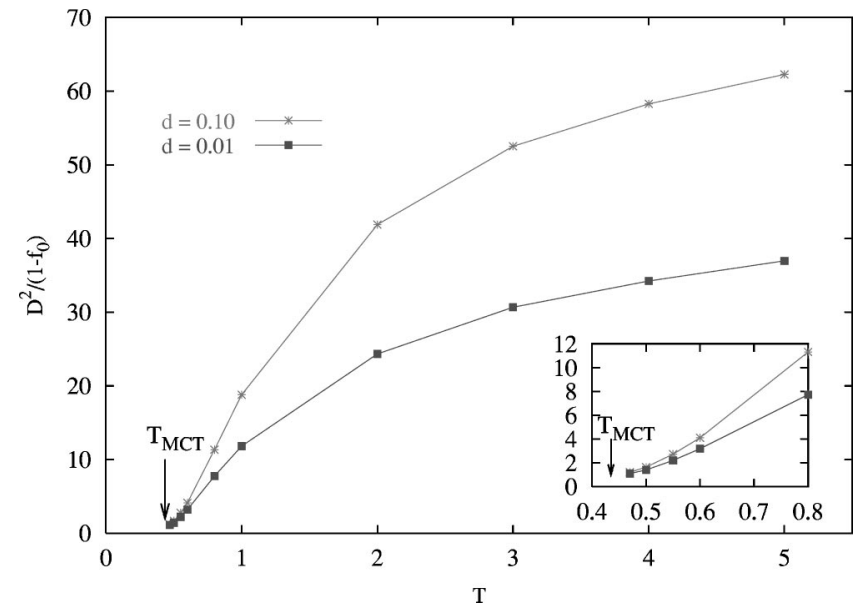

FIG. 1. Mean squared distance between inherent structures as a function of temperature for two different damages $d=0.1$ and 0.01 . Inset: a zoom of the low-temperature region.

landscape influenced region. Our results are an independent confirmation of the presence of that characteristic point but, more importantly, it gives a purely topological determination of it. From the inset it is apparent that the distance does not go to zero on approaching $T_{\mathrm{MCT}}$.

In order to get better insight into the landscape properties of the low-temperature region we show in Fig. 2 the probability of nonzero events as a function of temperature in $\log$-linear scale. It is evident the onset of a strong decrease in the non zero events at around $T=1$. The behavior of $1-f_{0}$ near $T_{\mathrm{MCT}}$ is obviously dependent on the initial damage. But even for the smallest damage $d=0.01$ (two orders of magnitude smaller than the typical interatomic distance) the number of nonzero events does not seem to go to zero at $T_{\mathrm{MCT}}$. In the inset of Fig. 2 we show $1-f_{0}$ in a reduced temperature range. The points below $T \simeq 0.6$ are perfectly aligned indicating an exponential decay of the number of nonzero events towards $T_{\mathrm{MCT}}$ of the form $1-f_{0}=\exp \left[\left(T-T_{0}\right) / \epsilon\right]$ with $T_{0}$ depending on the initial damage. We did several indepen-

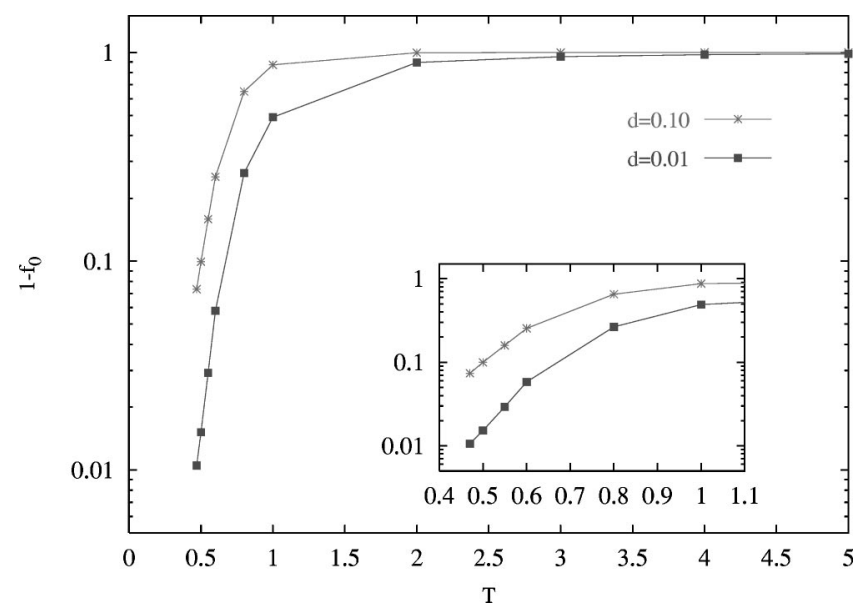

FIG. 2. The fraction of events with nonzero final distance as a function of temperature for two different damages in log-linear scale. Inset: a restricted temperature range in order to clearly show the alignment of points for $T \leqslant 0.6$. 


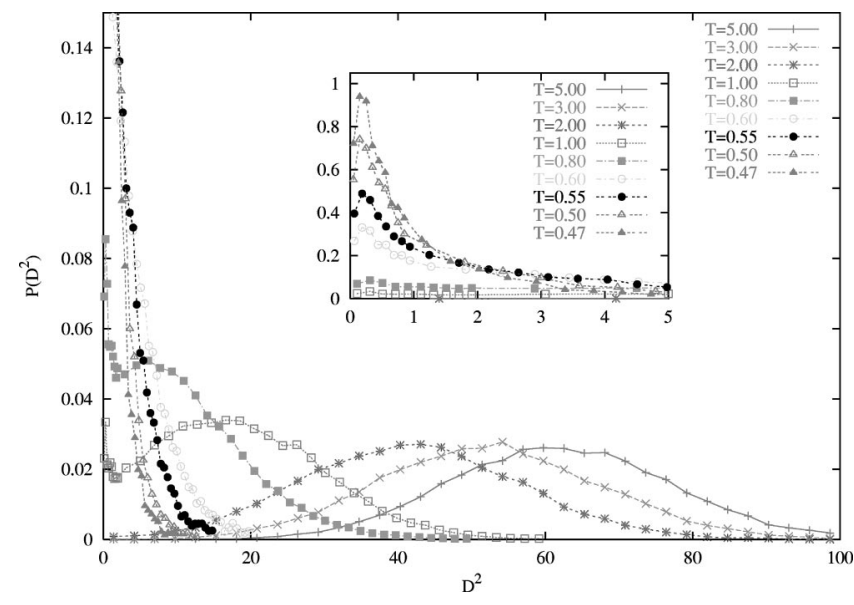

FIG. 3. The distribution of squared displacements contributing to the mean of Eq. (1) for the different temperatures studied for damage $d=0.10$. Note the presence of two maxima for $T=0.8$ and $T=1$. Inset: the peak of the distributions for very small displacements.

dent checks in order to confirm this behavior. The direct implication of this result is that the number of saddles does not vanish at $T_{\mathrm{MCT}}$ in agreement with recent work $[6,8]$ and in apparent contradiction with previous one [4]. As we have independently verified in our simulations that upon extrapolation to lower temperatures the diffusion constant should go to zero at $T_{\mathrm{MCT}}$, this, together with the previous result implies that there exist saddles at $T \leqslant T_{\mathrm{MCT}}$ associated with nondiffusional events.

One can get more direct evidence of the relation between the landscape topology and dynamics by looking directly at the particle displacements. The fact that particles cannot diffuse in a region where there is still minima-connecting saddles available may explain recent results obtained by Büchner et al. on the slowing down in the dynamics of supercooled liquids on approaching $T_{\mathrm{MCT}}$ [13]. In Ref. [13], the authors show that near $T_{\mathrm{MCT}}$ the system visits a kind of valley containing several IS where the system is trapped for some time jumping between the different IS and where the mobility is extremely small. Motion of particles in these valleys should be strongly collective and probably single displacements will not go beyond one interatomic distance. In order to test this hypothesis we have computed several displacement distribution functions.

In Fig. 3 we show the distribution of squared displacements, i.e., each value of $D^{2}$ contributing to this histogram is a sum over the 250 particles which corresponds to each term contributing to the mean squared displacement of Eq. (1). We see that at high temperatures $(T>1)$ the maximum is located at $D^{2}$ greater than 40 which is the number of particles perturbed. This indicates that an important fraction of the system suffer displacements at least of the order of the interatomic distance. An interesting fact emerges exactly at $T$ $=1$ and is also present at $T=0.8$ : a second maximum at very low values appears. This is another indication that $T=1$ is a characteristic temperature for this system below which the dynamics is landscape influenced as Sastry et al. named this region. In this crossover region three kinds of processes can

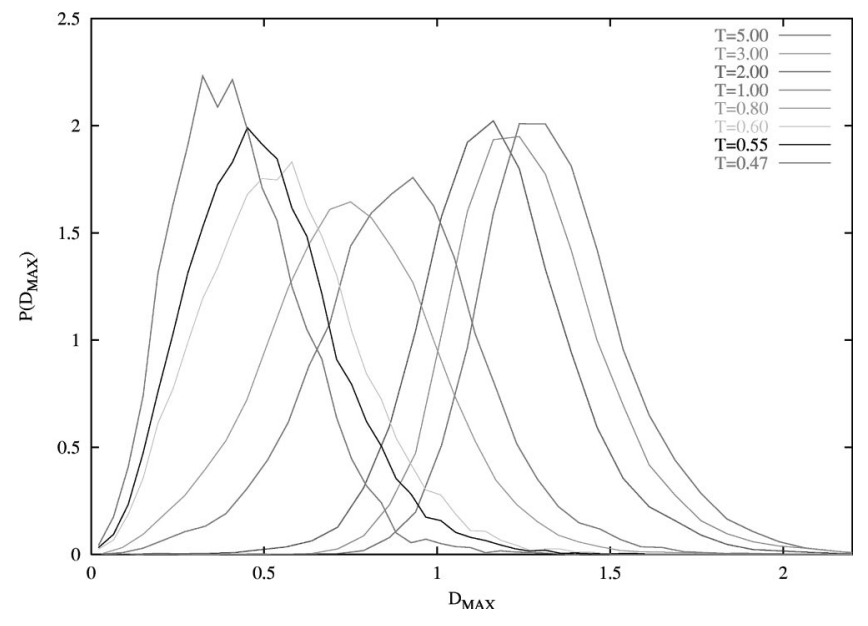

FIG. 4. The distribution of largest displacements for several temperatures for damage $d=0.10 . D_{\max }$ is the displacement of the particle that experiences the largest displacement of all the particles in the system in a given process. Note the large dispersion in the values at $T=0.8$ and $T=1$.

be detected: (i) processes in which a fraction of particles can still move by distances of the order of an interatomic distance, (ii) processes where the particles can only move by tiny amounts and there is no particle moving an interatomic distance, and (iii) a third group of processes where particles do not move at all. The last group contributes with a delta peak at the origin which we have eliminated from our plots. The growing confinement of the particles as the temperature is lowered from $T=1$ is more dramatic than what can be inferred from the growing peak at low values of the distance. In fact for $T<0.8$ there is only one peak at $D^{2}<0.5$ (see the inset of Fig. 3). If we note again that each contribution to $D^{2}$ is a sum over the 250 particles, this implies that on average no single particle moves a distance of the order of an interatomic distance. Of course, from the presence of the tails in the distributions for larger values of the distance one cannot discard a priori the existence of some particles that move by such amounts. Nevertheless it seems more likely that this contribution come essentially from collective motions in which an important number of the particles move very little. A stronger support for this scenario is given by the distribution of the largest displacements shown in Fig. 4.

This figure gives also very interesting information. Note that for $T>1$ the particle that moves more goes to a distance typically longer than one interatomic distance with very few contributions with $D_{\max }<1$. The variance of the distributions grow and the height of the peak goes through a minimum as the temperature crosses the region where the landscape begins to influence the dynamics. The large variance signals the appearance of an important fraction of particles which, although are the ones that move more than any other, have displacements confined to $D_{\max }<1$. Note that the distances between IS (Fig. 1) present an inflection point between $T$ $=0.8$ and $T=1$ indicating a rapid decrease in the number of saddles available for diffusing. For $T<0.8$ the maximum displacements are peaked around very small values less than one interatomic distance. 


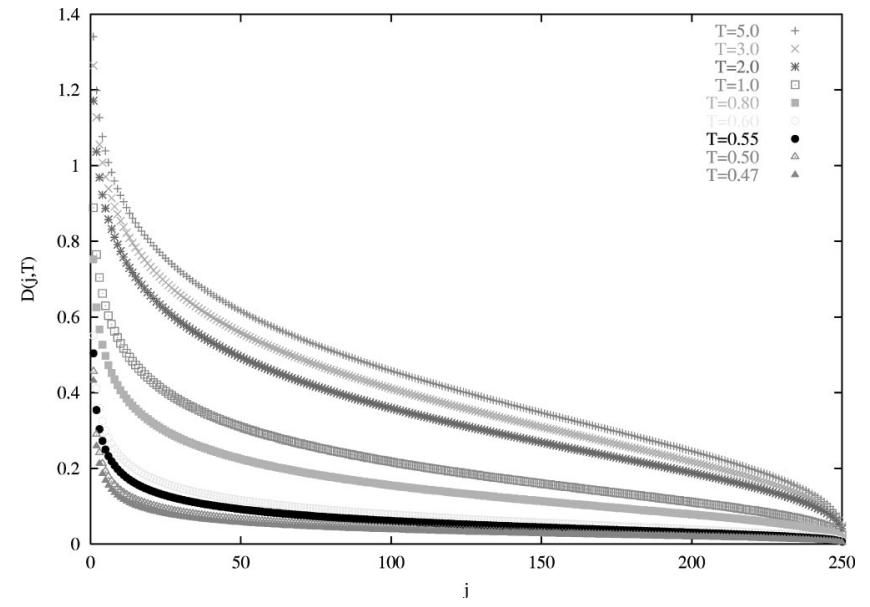

FIG. 5. The pattern of displacements for the 250 particles and for several temperatures.

Finally we asked the question: is there some order in the pattern of displacements per particle as a function of temperature? A partial but important answer can be done by ordering the $N$ particles by decreasing value of their displacements. In Fig. 5 we show that in fact the patterns of displacements are similar for all temperatures.

The first point to the left corresponds, for each temperature, to the mean over the particles with the largest displacements (first moment of distributions of Fig. 4), the second point is the mean over particles with the second largest displacements, and so on up to the particles with the smallest displacement. From this figure it is very clear that the overwhelming majority of particles move in average less than 1 while only very few move more than one interatomic distance at high temperatures $T>1$. For $T<0.8$ all particles in average move very little. Interestingly, the average distance traveled by the particles with the largest displacement goes to zero very slowly (almost linearly) as $T \rightarrow 0$ suggesting again a continuous variation of landscape properties on crossing $T_{\mathrm{MCT}}$. Also note that most particles move by some amount which means that the motion is highly collective. By looking at the relative difference between the first and second largest displacements (not shown) we obtained a practically constant and low value for $T>1$ followed by a pronounced grow of this difference for $T<1$ which is another indication of the drastic confinement of the system in this range of temperatures where only one particle moves much more than the others (although in a scale less than one interatomic distance).

Recently Grigera et al. [7] computed the distribution of particle displacements between a minimum and a saddle- connected neighbor in a soft spheres system and found evidence that, at low temperatures, the typical process is one where a large amount of particles travel very small distances, less than half an interatomic distance, and very few particles move by the order of one interatomic distance. These were interpreted as activated processes in a region where there were almost no saddles available. This picture is qualitatively different from the one that emerges from the present work for the binary LJ system. Nevertheless, further work is needed to elucidate if diffusion in a binary LJ system occurs as a cumulative effect of the small collective displacements or if it is due to very rare activated events that, if present, should be confined to the tails of our displacement distributions.

In summary we analyzed the information brought by the distances between near inherent structures relevant for the dynamics of a supercooled liquid near the mode coupling transition temperature. In this way the contributions coming from vibrations are automatically filtered out. It is possible to obtain rather precise information on the processes involved in the evolution in phase space and also in real space. The functional form of the MSD as a function of temperature and the evolution of the distribution of displacements and of maximum displacements define clearly a characteristic region near $T=1$ below which the number of saddles decays rapidly and the particles become strongly confined. We found also that the number of saddles is exponentially small on approaching the mode coupling transition temperature but does not go to zero. In the low-temperature region, in connecting two neighboring IS, most of the times all particles move by amounts much smaller than the typical interparticle distance. The scenario that emerges for the low-temperature dynamics is one in which the landscape is formed by a kind of metabasins $[6,8,14]$ in which there are still many inherent structures connected by low lying saddles which may exist even for $T<T_{\mathrm{MCT}}$ (see also the recent work by Heuer et al. [15]). Individual particles move by tiny amounts within a metabasin in a highly collective way. From our results on the binary LJ system the mode coupling transition temperature $T_{\mathrm{MCT}}$ continues to be characterized by a dynamical singularity and the possible connection of the transition itself with a sharp change in some topological property of the potential energy landscape remains to be elucidated.

We thank Toms Grigera and Andrea Cavagna for interesting comments. This work was partly supported by CONICET and Fundación Antorchas, Argentina, and by $\mathrm{CNPq}$ and FAPERGS, Brazil.
[1] P.G. Debenedetti and F.H. Stillinger, Nature (London) 410, 259 (2001).

[2] S. Sastry, P.G. Debenedetti, and F.H. Stillinger, Nature (London) 393, 554 (1998).

[3] F. Sciortino, W. Kob, and P. Tartaglia, Phys. Rev. Lett. 83, 3214 (1999).
[4] L. Angelani, R. Di Leonardo, G. Ruocco, A. Scala, and F. Sciortino, Phys. Rev. Lett. 85, 5356 (2000).

[5] K. Broderix, K.K. Bhattacharya, A. Cavagna, A. Zippelius, and I. Giardina, Phys. Rev. Lett. 85, 5360 (2000).

[6] T.F. Middleton and D.J. Wales, Phys. Rev. B 64, 024205 (2001). 
[7] T.S. Grigera, A. Cavagna, I. Giardina, and G. Parisi, Phys. Rev. Lett. 88, 055502 (2002).

[8] J.P.K. Doye and D. Wales, J. Chem. Phys. 116, 3777 (2002).

[9] W. Götze and L. Sjögren, Rep. Prog. Phys. 55, 241 (1992).

[10] W. Kob and H.C. Andersen, Phys. Rev. E 51, 4626 (1995).

[11] W. Kob and H.C. Andersen, Phys. Rev. Lett. 73, 1376 (1994).

[12] The particles to be displaced are chosen randomly for $i$
$=1, \ldots, n_{d} / 2$ and the other $n_{d} / 2$ are chosen so that $\delta \mathbf{r}_{i}=$ $-\delta \mathbf{r}_{i-n_{d} / 2}$ in order to preserve the center of mass of the system.

[13] S. Büchner and A. Heuer, Phys. Rev. Lett. 84, 2168 (2000).

[14] F.H. Stillinger, Science 267, 1935 (1995).

[15] B. Doliwa and A. Heuer, e-print cond-mat/0205283. 